\title{
COMPRESSION ALgORITHM SELECTION FOR Multispectral MASTCAM IMAgES
}

\author{
Chiman Kwan, Jude Larkin, Bence Budavari, and Bryan Chou \\ Applied Research, LLC, Rockville, Maryland, USA
}

\begin{abstract}
The two mast cameras (Mastcam) onboard the Mars rover, Curiosity, are multispectral imagers with nine bands in each camera. Currently, the images are compressed losslessly using JPEG, which can achieve only two to three times compression. We present a two-step approach to compressing multispectral Mastcam images. First, we propose to apply principal component analysis (PCA) to compress the nine bands into three or six bands. This step optimally compresses the 9-band images through spectral correlation between the bands. Second, several well-known image compression codecs, such as JPEG, JPEG-2000 (J2K), X264, and X265, in the literature are applied to compress the 3-band or 6-band images coming out of PCA. The performance of different algorithms was assessed using four well-known performance metrics. Extensive experiments using actual Mastcam images have been performed to demonstrate the proposed framework. We observed that perceptually lossless compression can be achieved at a 10:1 compression ratio. In particular, the performance gain of an approach using a combination of PCA and X265 is at least $5 \mathrm{dBs}$ in terms peak signal-to-noise ratio (PSNR) at a 10:1 compression ratio over that of JPEG when using our proposed approach.
\end{abstract}

\section{KEYWORDS}

Perceptually lossless compression; Mastcam images; multispectral images; JPEG; JPEG-2000; X264; $X 265$

\section{INTRODUCTION}

The Mars rover, Curiosity, has many instruments onboard for Mars data collection and in-situ surface characterization [1]. Alpha Particle X-ray Spectrometer (APXS) [2], Laser Induced Breakdown Spectrometer (LIBS) [3][4], and Mastcam [5]-[8] are well-known ones. Quite a few of these instruments are imagers that fight for limited bandwidth to transmit data back to Earth. Currently, the Mastcam images are all compressed using JPEG, a technology developed during the 1990's [9]. Although JPEG is simple and efficient, the compression ratio can be, at most, between two to three times. There are new compression standards developed in the past two decades. Well-known video codecs include X264 [10] and X265 [11], which are also applicable to still image compression. X264 and X265 also provide lossless compression options. In some applications such as security monitoring in shopping centers or home surveillance, people are still using lossless image compression algorithms (JPEG [9] and J2K [12]) for compressing videos frame by frame. This practice may be too conservative, as lossless compression can only achieve two to three times compression.

In an earlier work, we focused on the compression of only the RGB Mastcam images with only three bands [8]. In this research, we aim at achieving perceptually lossless compression with a 10:1 compression ratio for multispectral Mastcam images. We propose to apply a new compression framework for Mastcam images that compresses in both the spectral and spatial domain. First, we propose to apply PCA [13] to compress nine bands into three or six bands in the PCA domain. According to theory, PCA achieves optimal compression in this step. Other spectral 
compression techniques such as discrete cosine transform (DCT), fast Fourier transform (FFT), or wavelet transforms are all sub-optimal. Second, well-known codecs (JPEG, X264, X265 and J2K) are then applied to compress the PCA bands. Four performance metrics were used in our study. Two of them are motivated by the human visual system and hence are suitable for judging perceptually lossless image quality. Extensive experiments have been performed using actual Mastcam images. It was observed that the combination of PCA and X265 yielded the best performance. Most importantly, the performance gain is at least $5 \mathrm{dBs}$ in terms PSNR at a 10:1 compression ratio over JPEG when using our proposed approach.

It is emphasized here that our paper is an application oriented paper, focusing on a niche application that no one has done a systematic study before. Although some of the compression techniques used in this paper are well-known, we still would like to stress two key contributions of our research. The first key contribution of our project is to integrate PCA and conventional image codecs into a unified framework for compressing Mastcam images. Although PCA has been combined with J2K in [19], this does not mean that the PCA and J2K combination is the best for our application, as every application is different. One should not blindly use the combination of PCA and $\mathrm{J} 2 \mathrm{~K}$ for a new application without some thorough investigations. It turns out that, based on our extensive studies in this paper, we found that the combination of PCA and $\mathrm{X} 265$ is actually the best performing one. The second key contribution is that, to the best of our knowledge, we are the first ones to investigate the possibility of replacing JPEG with some advanced compression schemes for Mastcam images. Based on our investigations so far, it appears that a newer compression framework may need to be used in compressing Mastcam images, as more images can be transmitted with the same bandwidth.

Our paper is organized as follows. Section 2 summarizes the technical approach and its components. Section 3 summarizes all the experiments using actual Mastcam images. Finally, concluding remarks will be given in Section 4.

\section{TECHNiCAL APPROACH}

\subsection{Proposed Multispectral Image Compression Framework}

Our overall technical approach can be summarized in Figure 1. First, we apply PCA to compress the 9-band image into three or six bands in the PCA domain. This step is somewhat similar to wavelet or fast Fourier transform (FFT), as the image pixels are transformed to other domains. According to theory, PCA achieves optimal compression as compared to other transformations. Second, we apply some conventional image codecs such as JPEG, J2K, X264, and X265 to compress the three or six PCA bands. After this 2-step compression, the file size will be compared to the original file size to generate the compression ratio. To evaluate the quality of the reconstructed images, an inverse compression process needs to be performed where we first decompress the images using the conventional codecs and then an inverse PCA is performed to reconstruct the nine bands. The reconstructed bands will be compared to the uncompressed nine bands to generate the various performance metrics.

Although there are some other compression codecs such as VP8 [14] and VP9 [15] in the literature, we used the most popular ones in the literature where open source codes are available. We briefly describe some of the components shown in Figure 1 below.

- PCA [13] - PCA has been widely used in many applications, including sensor network data compression and hyperspectral image compression. The idea is to perform a singular value decomposition (SVD) to the original 9-band image. The first 
Signal \& Image Processing: An International Journal (SIPIJ) Vol.10, No.1, February 2019

three or six principal components are retained. PCA has the optimal compression performance than others [16]-[21].

- JPEG [9] - JPEG is the very first image compression standard which was initially released in 1992. The video counterparts are the MPEG-1 and MPEG-2 standards.

- X-264 [10] - X264 is the current state-of-the-art in video compression. Youtube, Facebook, etc. all use X264. It also has good performance for still image compression.

- X-265 [11] - This is the next-generation video codec and has excellent still image compression and video compression. However, the computational complexity is much more than that of X264.

- J2K [12] - Unlike JPEG, X264, and X265, which all use DCT, J2K is wavelet based [22]-[25]. It was initially released in 2000.

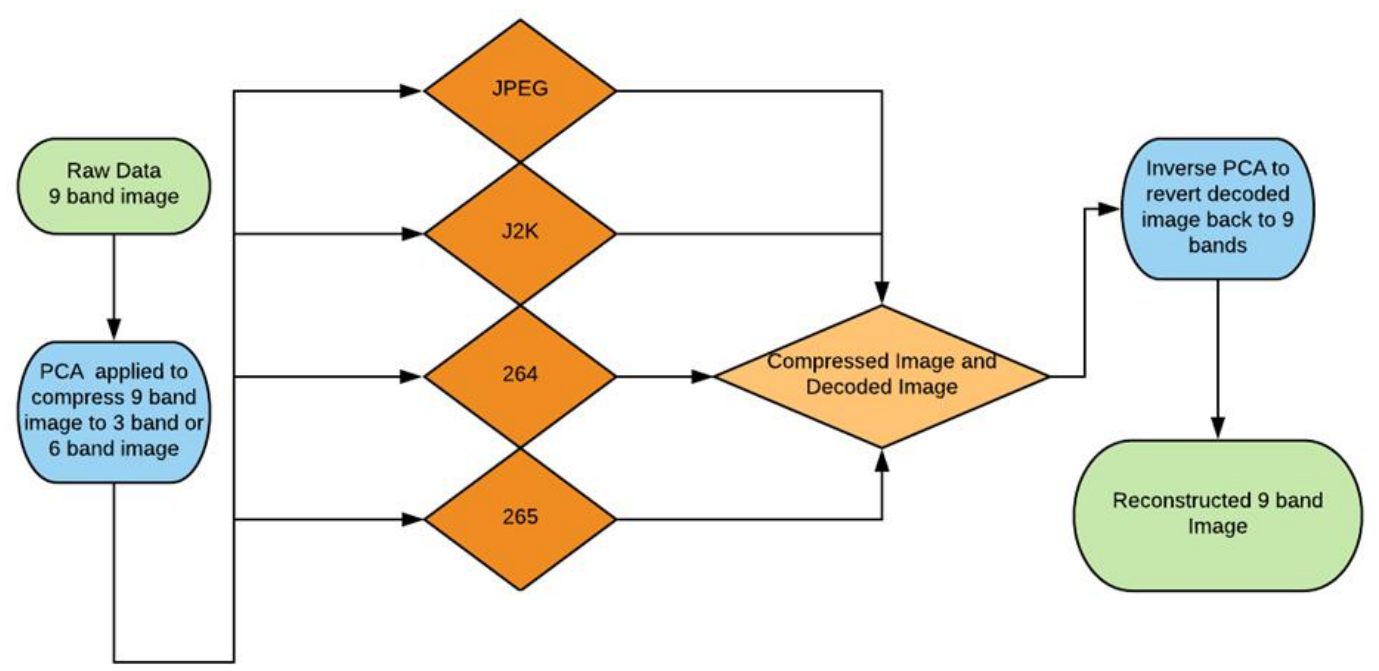

Figure 1. Proposed 2-step compression scheme for Mastcam images.

\subsection{Performance Metrics}

In almost all compression systems, researchers used PSNR or structural similarity (SSIM) to evaluate the compression algorithms. Given a fixed compression ratio, algorithms that yield higher PSNR or SSIM will be regarded as better algorithms. However, PSNR or SSIM do not correlate well with human perception. Recently, a group of researchers investigated a number of different performance metrics [26] that were motivated from human visual system (HVS). Extensive experiments were performed to investigate the correlation between human perception with various performance metrics. According to the findings found in [26], it was determined that two performance metrics (HVS and HVS-m) correlate well with human perception. Details can be found in [26]. Hence, in addition to PSNR and SSIM, we also used HVS and HVS-m for assessing perceptually lossless compression with respect to Mastcam images. For completeness, we include a figure from [26] that demonstrates that HVS yields better correlation with human perception. In the past, we have used HVS and HVS-m in several applications [8][27][28]. 

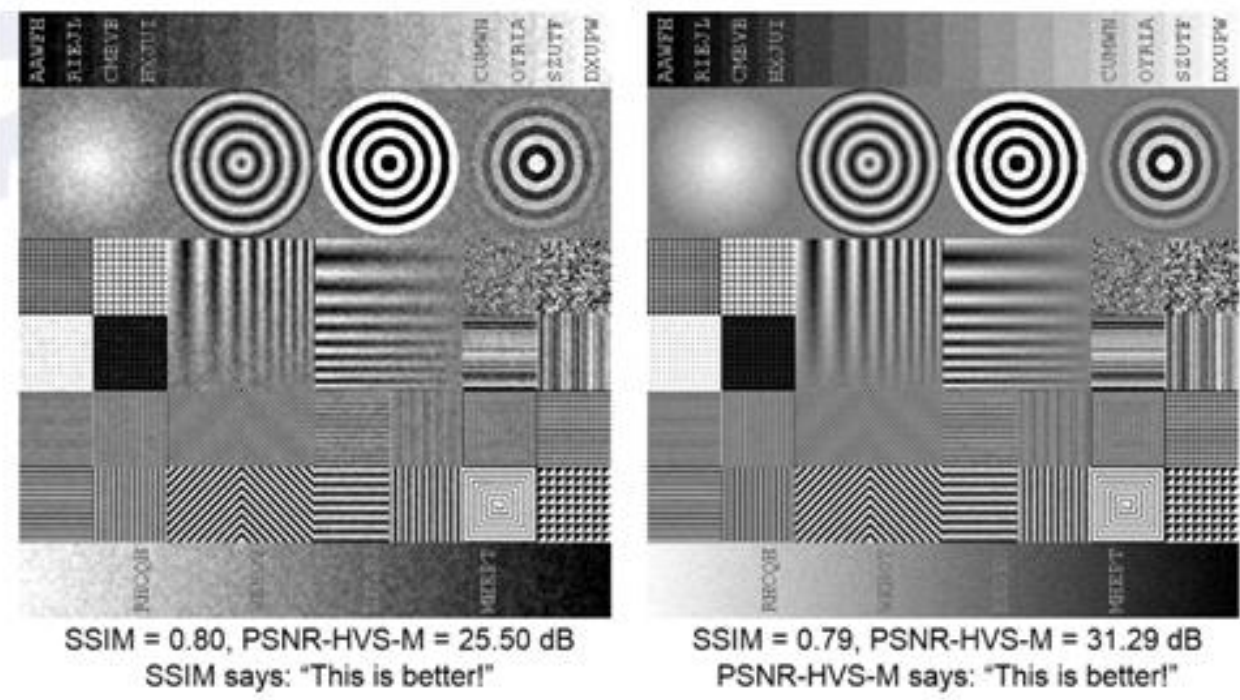

Figure 2. Comparison of SSIM and HVS-m. HVS-m has better correlation with human perception [26].

\section{EXPERIMENTAL RESULTS}

\subsection{Mastcam Imager and Mastcam Images}

Mastcam imager information is shown in Figure 3 and Table 1. There are six overlapping bands and three non-overlapping bands (L3, L4 and L5 from the left camera and R3, R4, and R5 from the right camera). More details about Mastcam can be found in [1]. Figure 4 and Figure 5 show 16 left Mastcam images and 15 right Mastcam images, respectively.

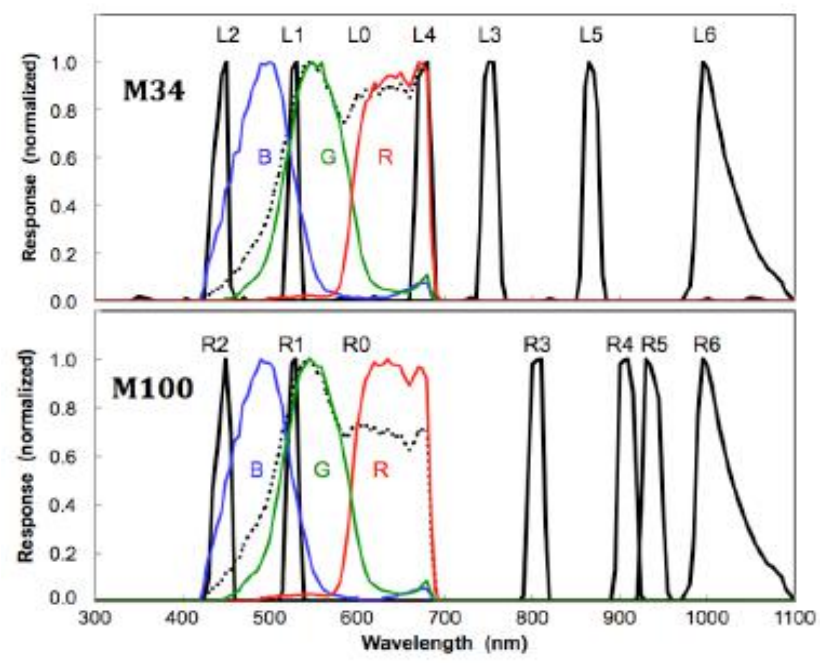

Figure 3. Normalized MSL/Mastcam system-level spectral response profiles for the left eye M-34 camera (top panel) and the right eye M-100 camera (bottom panel) [1].

Table 1. Mastcam filters in the order of increasing wavelengths [1]. 
Signal \& Image Processing: An International Journal (SIPIJ) Vol.10, No.1, February 2019

\begin{tabular}{|c|c|c|c|}
\hline \multicolumn{2}{|c|}{ The left Mastcam (M-34) } & \multicolumn{2}{c|}{ The right Mastcam (M-100) } \\
\hline Filter & Wavelength $\lambda \pm H W H M(n m)$ & Filter & Wavelength $\lambda \pm H W H M(n m)$ \\
\hline \hline L2 & $445 \pm 10$ & R2 & $447 \pm 10$ \\
\hline L0B & $495 \pm 37$ & R0B & $493 \pm 38$ \\
\hline L1 & $527 \pm 7$ & R1 & $527 \pm 7$ \\
\hline L0Gb & $554 \pm 38$ & R0Gb & $551 \pm 39$ \\
\hline L0R & $640 \pm 44$ & R0R & $638 \pm 44$ \\
\hline L4 & $676 \pm 10$ & R3 & $805 \pm 10$ \\
\hline L3 & $751 \pm 10$ & R4 & $908 \pm 11$ \\
\hline L5 & $867 \pm 10$ & R5 & $937 \pm 11$ \\
\hline L6 & $1012 \pm 21$ & R6 & $1013 \pm 21$ \\
\hline
\end{tabular}

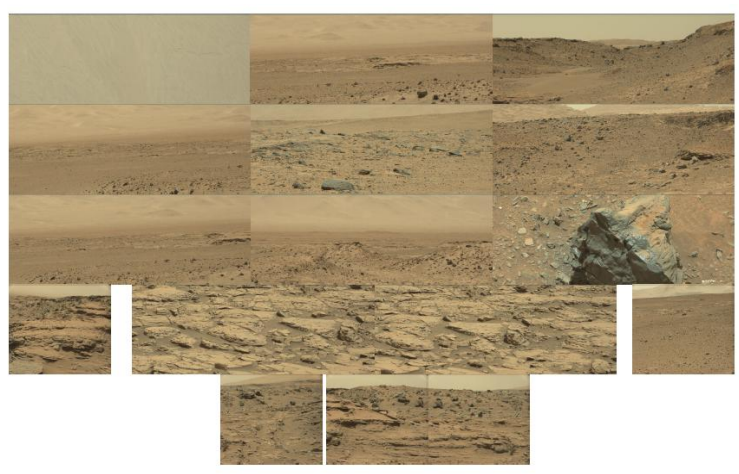

Figure 4. Images from the left Mastcam.

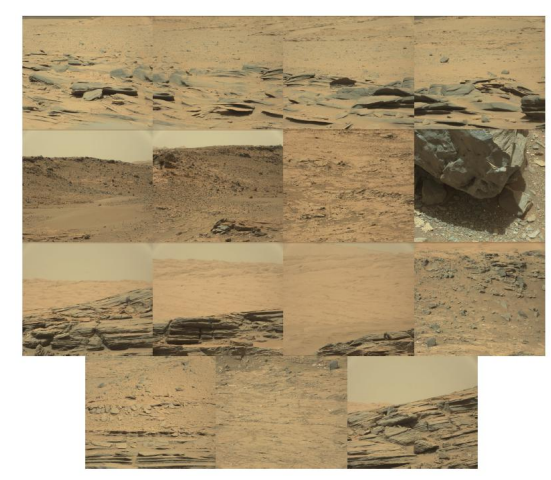

Figure 5. Images from the right Mastcam.

\subsection{Compression Results}

For the proposed 2-step approach, we tested 2 different cases: 3-band PCA and 6-band PCA. In both cases, we first applied PCA to the original 9-band Mastcam images and then proceeded to apply the various conventional compression methods.

\subsubsection{PCA-3}

From Figure 6 and Figure 7, one can see that the J2K, X264, and X265 all perform very closely in all four of the metrics generated for the PCA to 3-band approach. It should be noted that we used compression ratio rather than bits per pixel because we take the image overhead information into account and hence it is more close to actual practice. X265 performs better in all available metrics. X264 also performs closely to the X265 in all metrics around the $0.1-0.16$ compression ratios. It even slightly outperformed the X265 in HVS and HVS-m at those compression ratios. It should be emphasized that JPEG is far worse than the other methods. 
Signal \& Image Processing: An International Journal (SIPIJ) Vol.10, No.1, February 2019

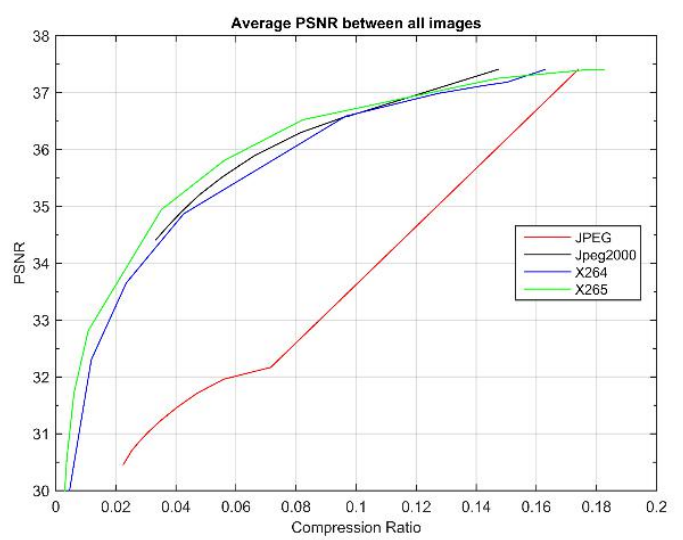

(a)

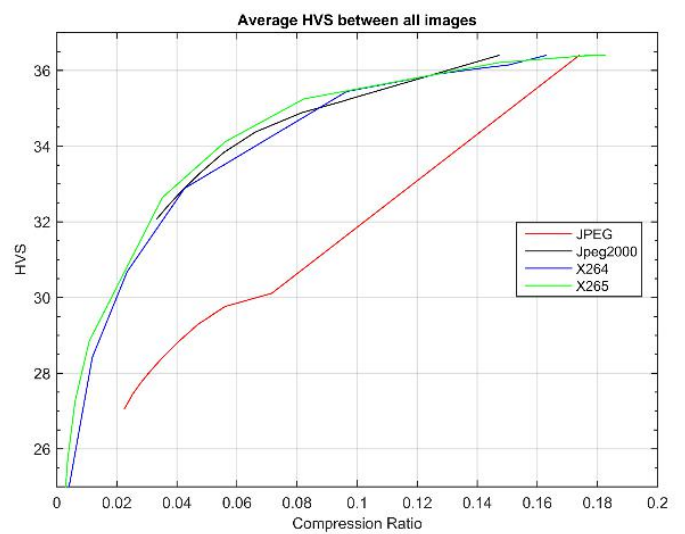

(c)

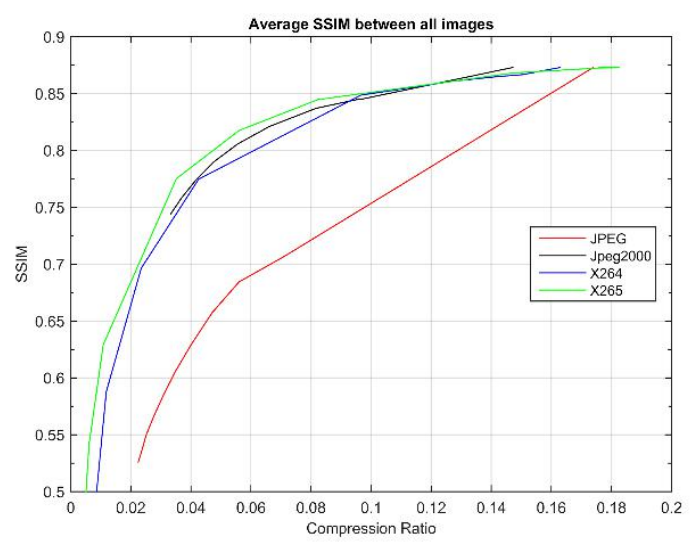

(b)

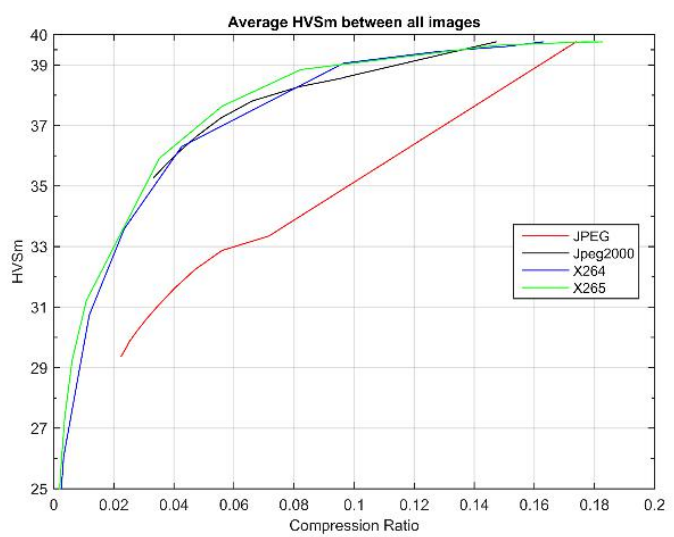

(d)

Figure 6. PCA-3: Comparison of different algorithms for 20 left Mastcam mages shown in Figure 4: (a) PSNR in dB for left images; (b) SSIM for left images; (c) HVS in dB for left images; (d) HVSm in dB for left images.

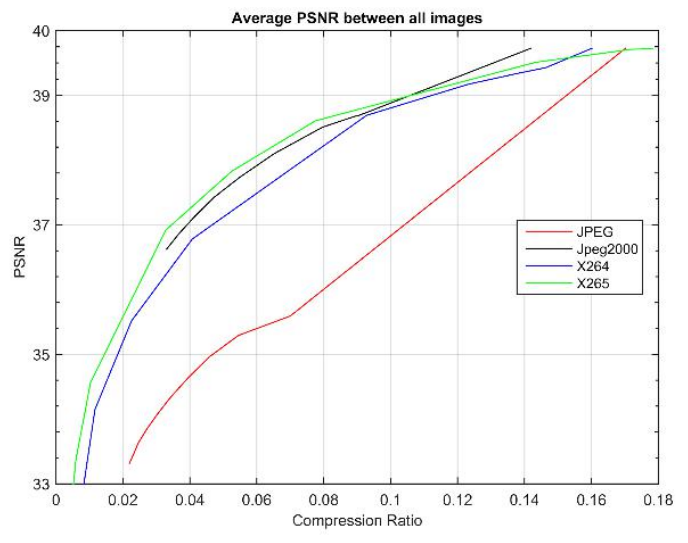

(a)

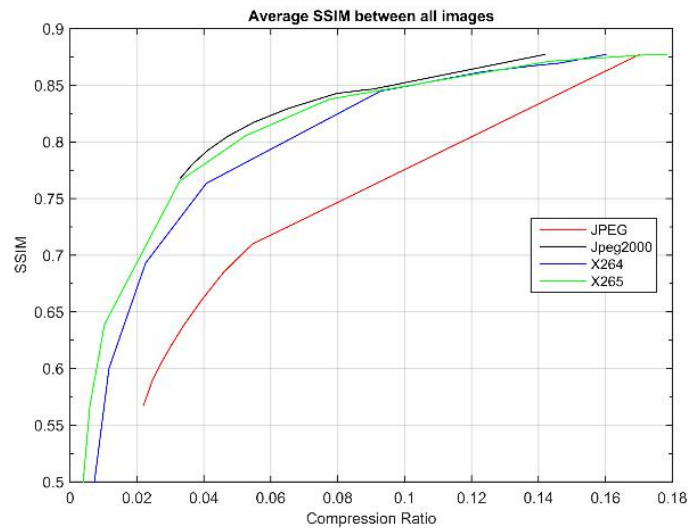

(b) 


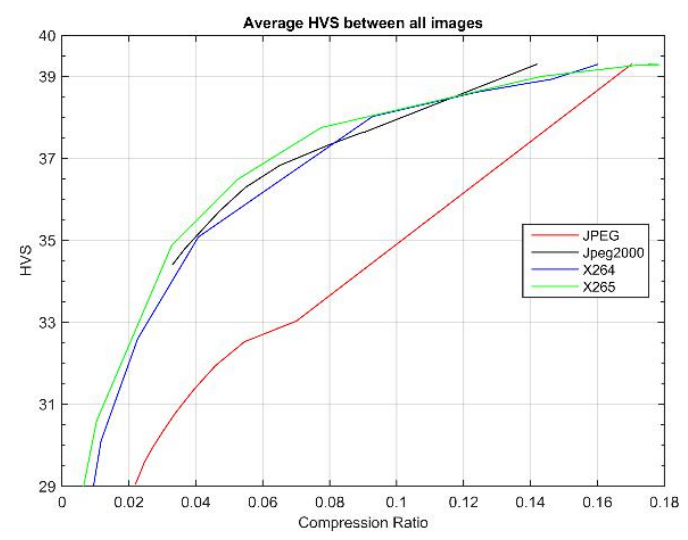

(c)

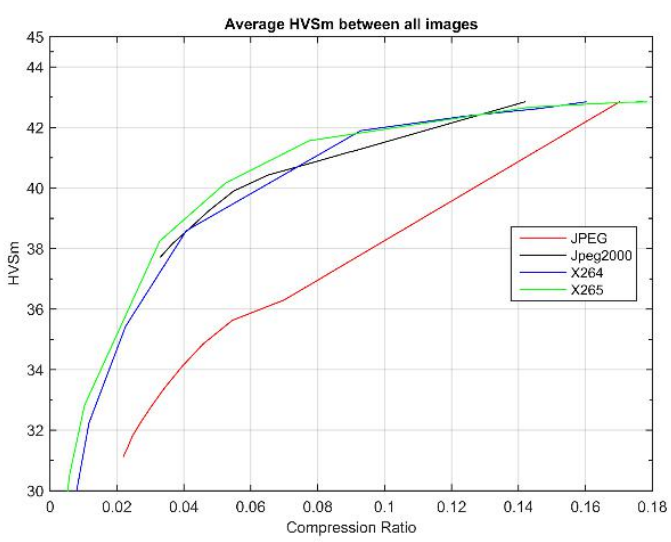

(d)

Figure 7. PCA-3: Comparison of different algorithms for 20 right Mastcam images shown in Figure 5: (a) PSNR in $\mathrm{dB}$ for right images; (b) SSIM for right images; (c) HVS in dB for right images; (d) HVSm in dB for right images.

\subsubsection{PCA-6}

The compression results for PCA-6 are shown in Figure 8 and Figure 9. When applying PCA to generate six bands, the methods had similar relative performance between each other, which is similar to the PCA-3 case. The one noticeable difference between the 6-band and 3-band approach is that X265 and $\mathrm{J} 2 \mathrm{~K}$ are much closer to each other in all metrics. In the PCA-3 approach excluding the lossless case, X265 outperformed J2K in almost all metrics. For this 6band approach, J2K, however, has slightly better HVS and HVSm at compression ratios of 0.05 to 0.1 in some cases. Similar to the PCA-3 case, JPEG is at least $5 \mathrm{dBs}$ lower than other in terms of PSNR.

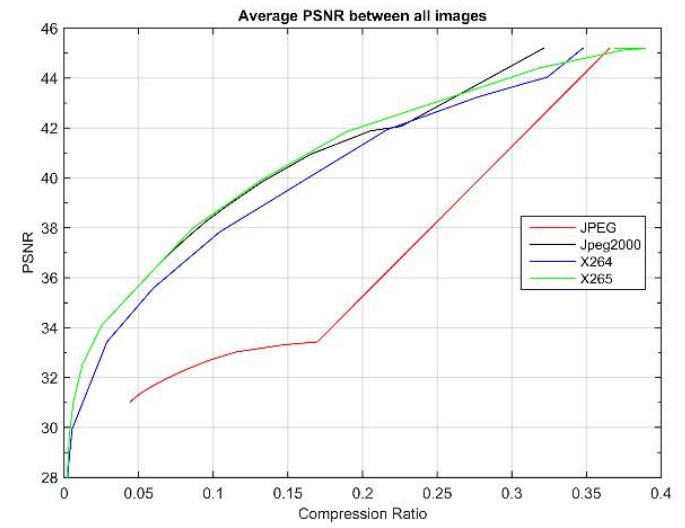

(a)

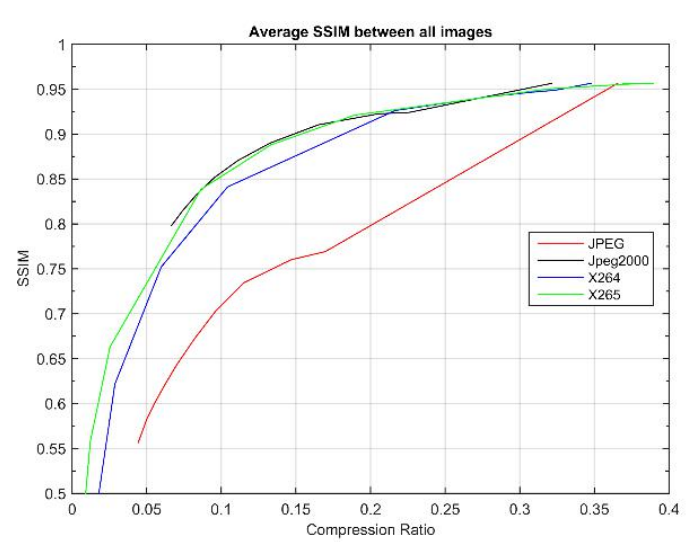

(b) 
Signal \& Image Processing: An International Journal (SIPIJ) Vol.10, No.1, February 2019

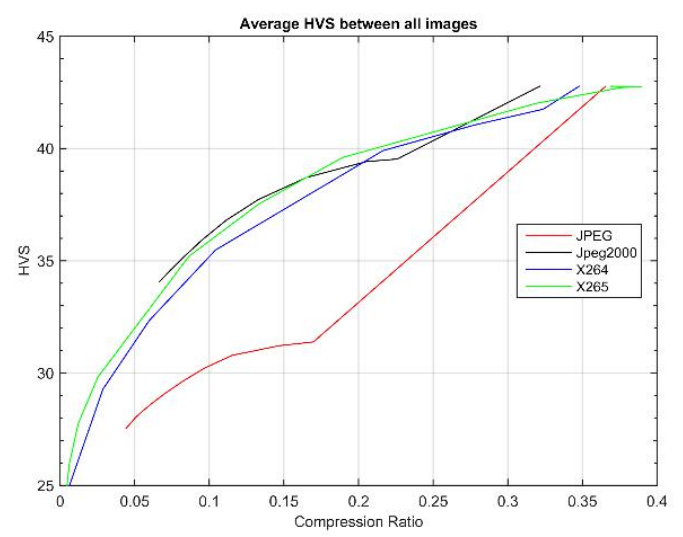

(c)

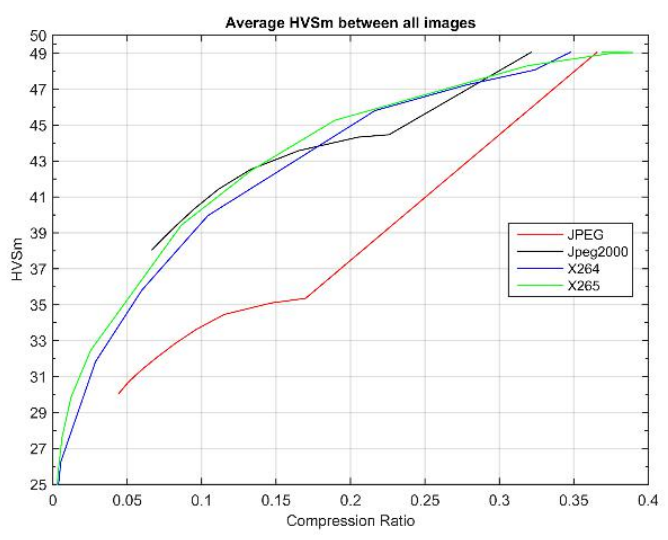

(d)

Figure 8. PCA-6: Comparison of different algorithms for 20 left images shown in Figure 4: (a) PSNR in dB for left images; (b) SSIM for left images; (c) HVS in dB for left images; (d) HVSm in dB for left images.

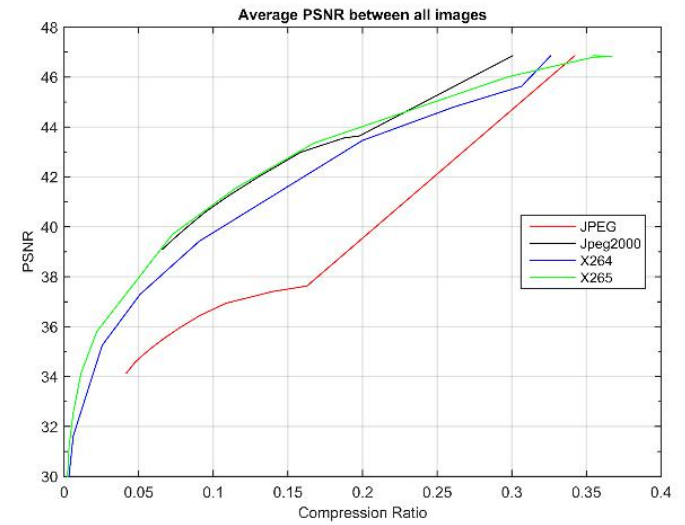

(a)

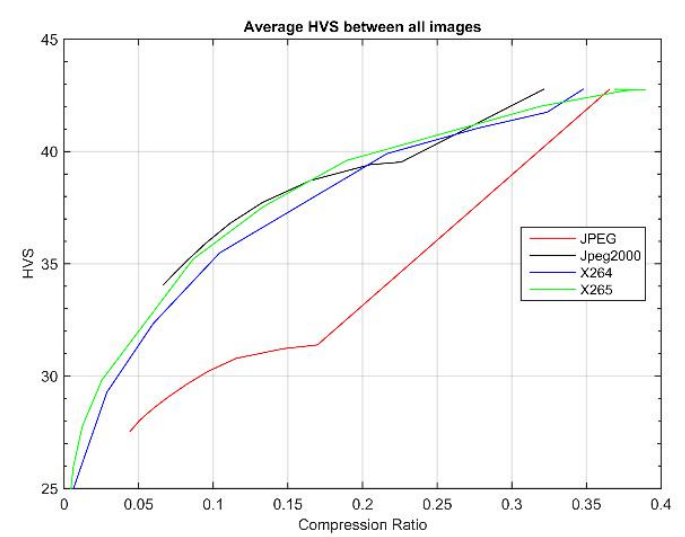

(c)

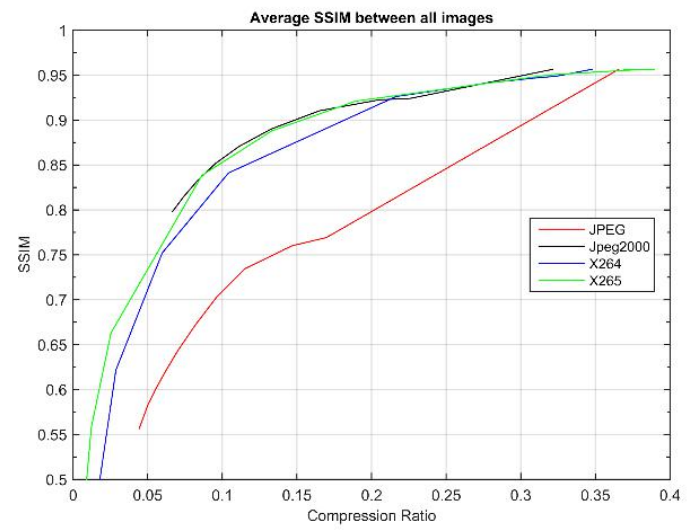

(b)

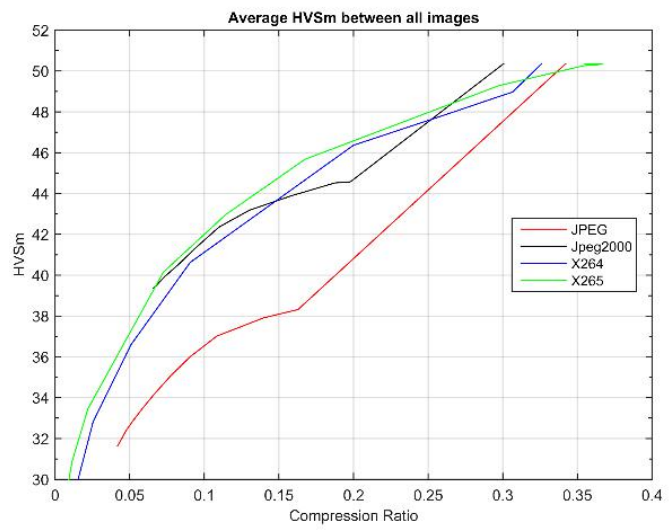

(d)

Figure 9. PCA-6: Comparison of different algorithms for 20 right images shown in Figure 5: (a) PSNR in $\mathrm{dB}$ for right images; (b) SSIM for right images; (c) HVS in dB for right images; (d) HVSm in dB for right images. 


\subsubsection{Comparison of PCA-3 and PCA-6}

The PCA-6 case had better results for all methods from 0.1 to 0.2 compression rates corresponding to 10 to 5 times compression, respectively. This can be seen in Figure 10 to Figure 13. At 0.1 compression ratio (Figure 10 and Figure 11), the combinations of PCA-6 and X265, and PCA-6 and J2K have very close performance. For left Mastcam images (Figure 10), the combination of PCA-6 and J2K has better performance in SSIM, HVS, and HVSm. For right images (Figure 11), the results are mixed. The performance of JPEG is the worst. Most importantly, the improvement of the best compression algorithm (X265) is about $5 \mathrm{dBs}$ in terms of PSNR (Figure 10(a) and Figure 11(a)) better than JPEG at 0.1 compression ratio. At 0.2 compression ratio (Figure 12 and Figure 13), the combination of PCA and X265 is overwhelmingly better than other combinations. The improvement of the best compression algorithm (X265) is about $7 \mathrm{dBs}$ in terms of PSNR (Figure 12(a) and Figure 13(a)) better than JPEG at 0.2 compression ratio. Thus, we think that it is about time to recommend to NASA about adopting a new approach to Mastcam image compression.

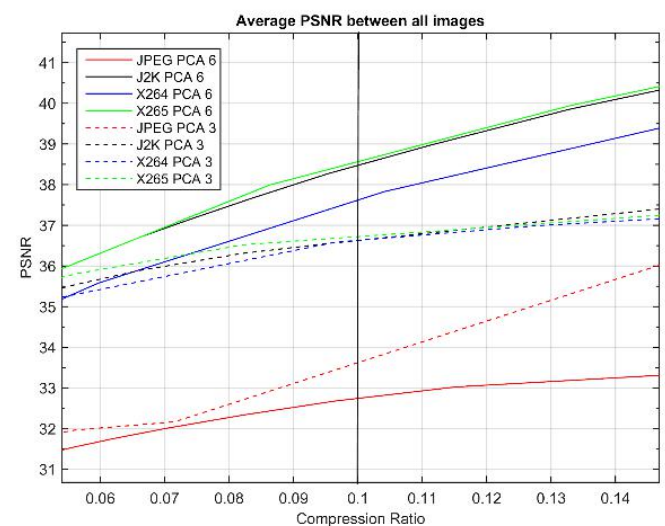

(a)

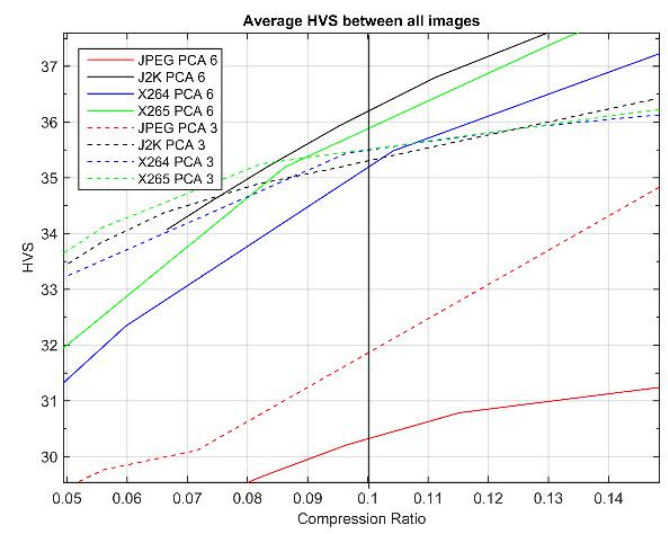

(c)

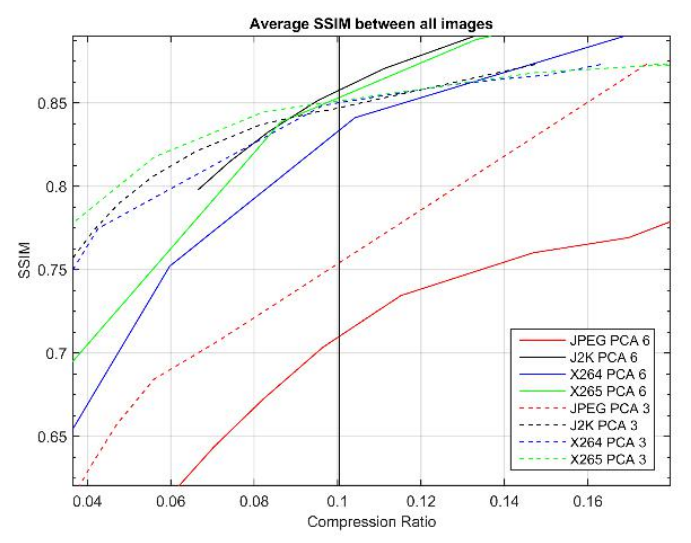

(b)

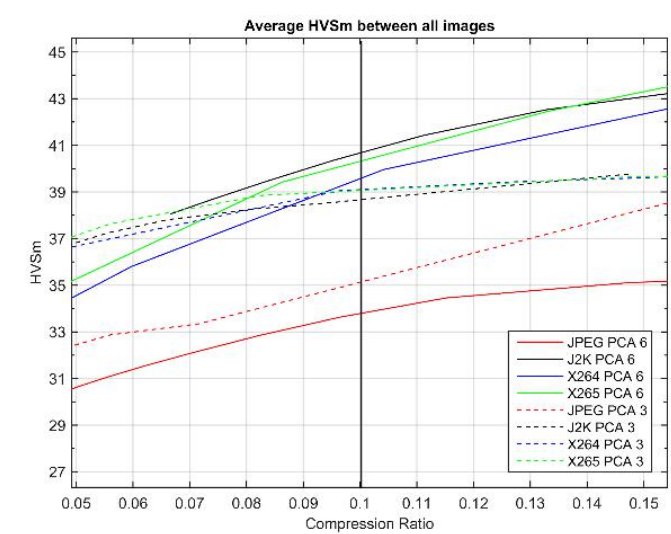

(d)

Figure 10. Comparison of PCA-3 and PCA-6 for left Mastcam images. Region of interest: compression ratio of 0.1: (a) PSNR in dB for left images; (b) SSIM for left images; (c) HVS in dB for left images; (d) HVSm in $\mathrm{dB}$ for left images. 
Signal \& Image Processing: An International Journal (SIPIJ) Vol.10, No.1, February 2019

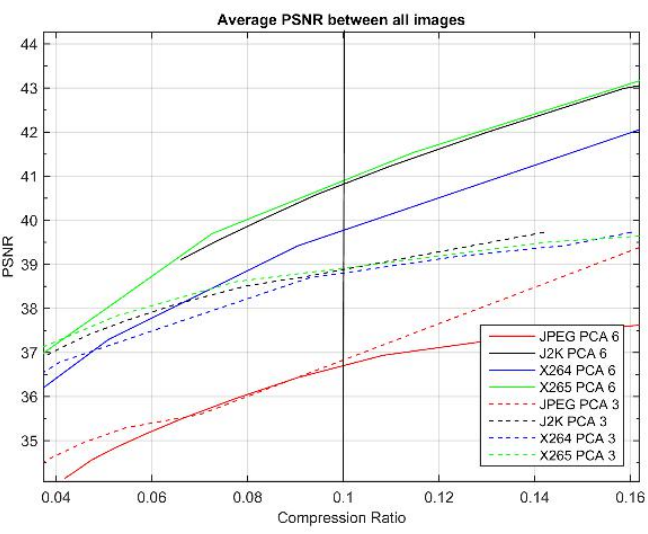

(a)

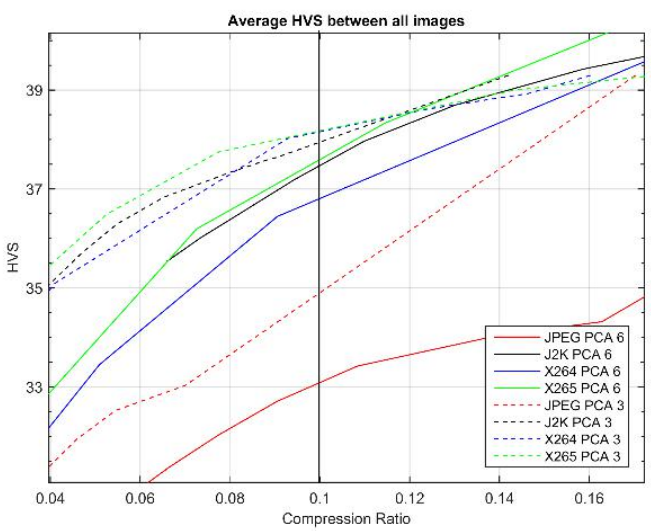

(c)

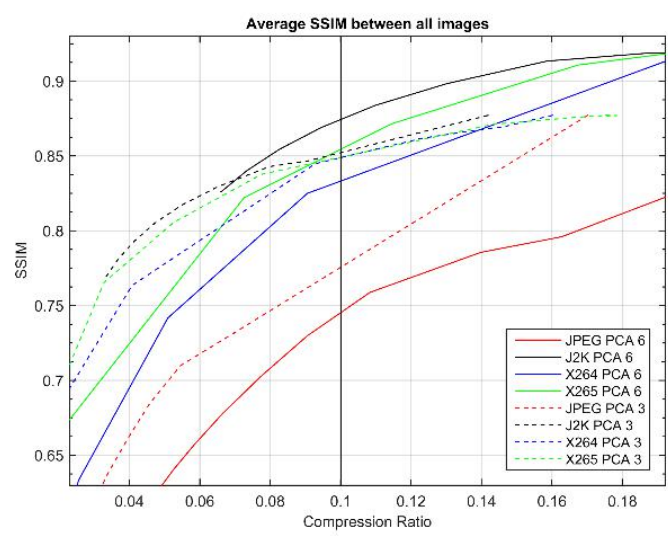

(b)

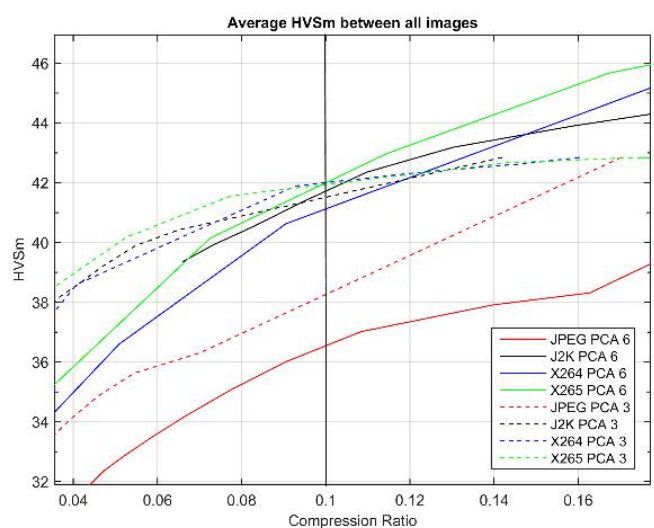

(d)

Figure 11. Comparison of PCA-3 and PCA-6 for right Mastcam images. Region of interest: compression ratio of 0.1: (a) PSNR in $\mathrm{dB}$ for right images; (b) SSIM for right images; (c) HVS in $\mathrm{dB}$ for right images; (d) HVSm in $\mathrm{dB}$ for right images

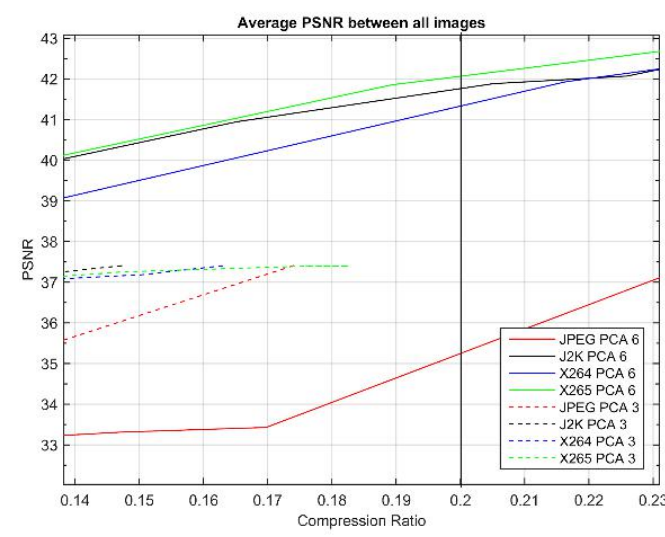

(a)

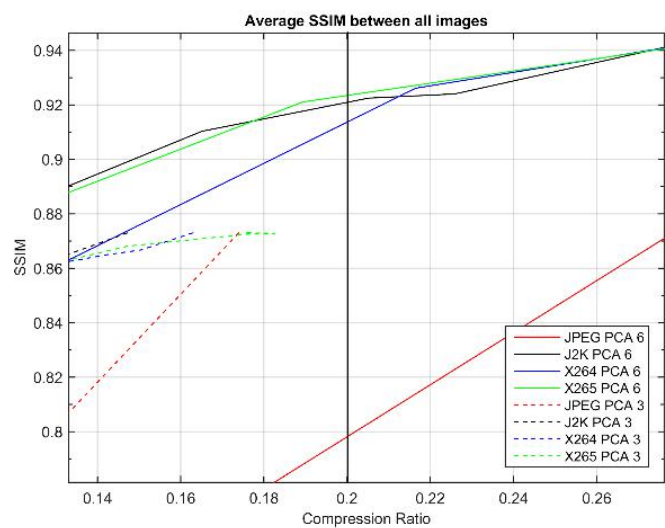

(b) 
Signal \& Image Processing: An International Journal (SIPIJ) Vol.10, No.1, February 2019

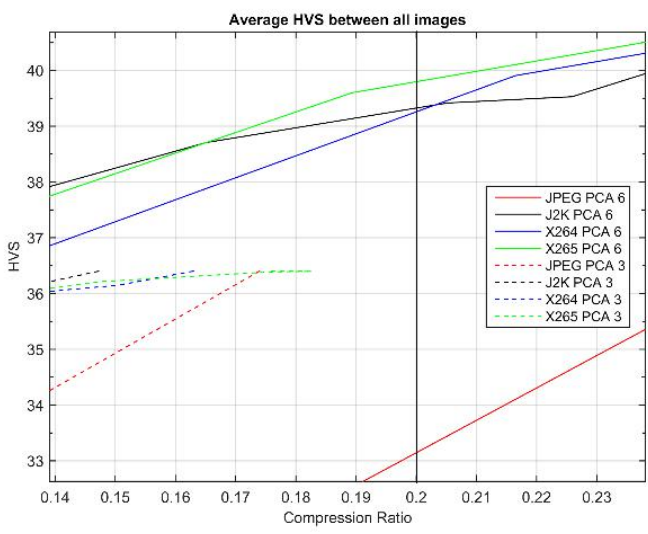

(c)

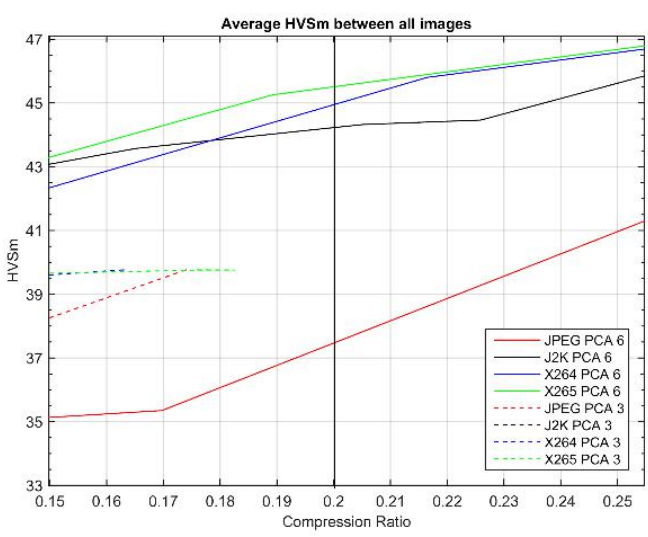

(d)

Figure 12. Comparison of PCA-3 and PCA-6 for left Mastcam images. Region of interest: compression ratio of 0.2: (a) PSNR in $\mathrm{dB}$ for left images; (b) SSIM for left images; (c) HVS in dB for left images; (d) HVSm in dB for left images

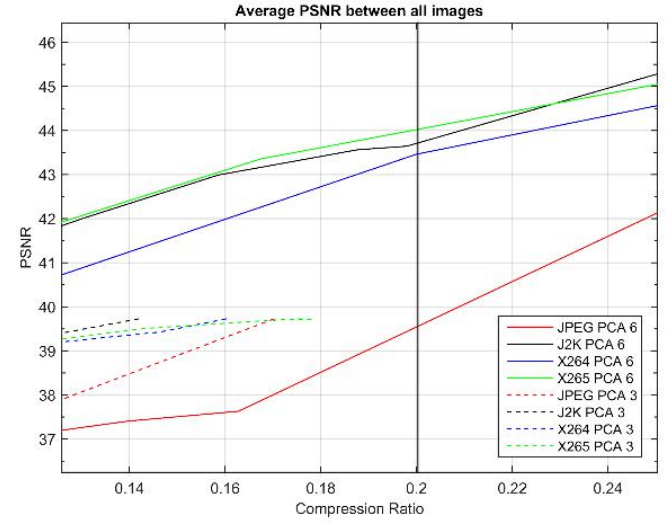

(a)

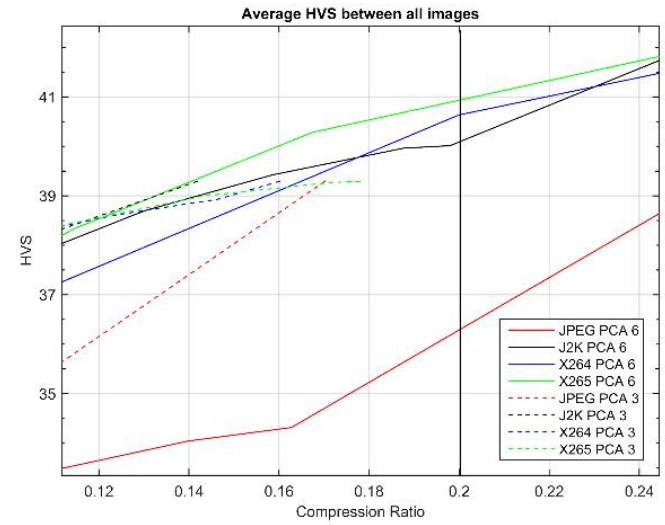

(c)

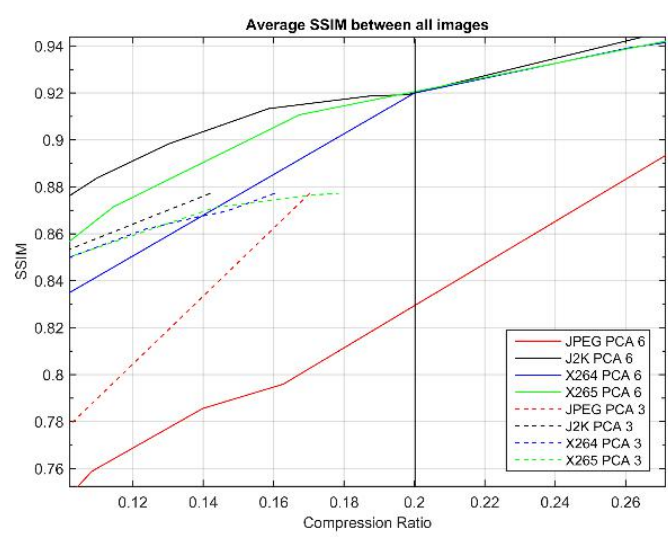

(b)

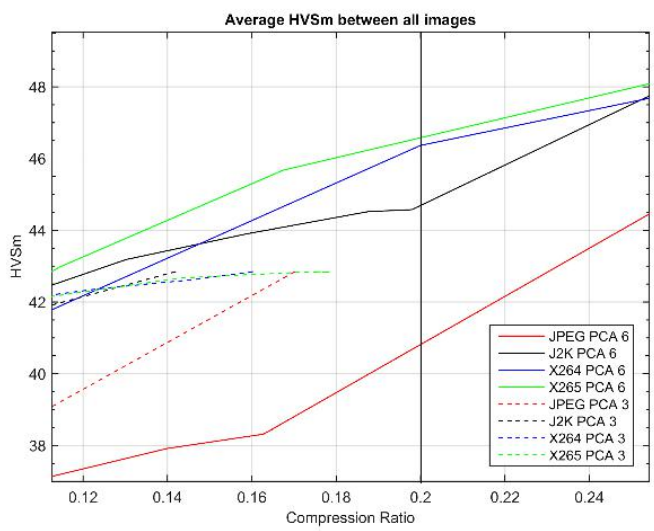

(d)

Figure 13. Comparison of PCA-3 and PCA-6 for right Mastcam images. Region of interest: compression ratio of 0.2: (a) PSNR in $\mathrm{dB}$ for right images; (b) SSIM for right images; (c) HVS in $\mathrm{dB}$ for right images; (d) HVSm in $\mathrm{dB}$ for right images. 


\section{Conclusions}

In this research, we investigated the performance of an alternative approach to compressing multispectral Mastcam images. The goal is to see if it is time to replace JPEG with newer and better compression algorithms for NASA applications. We have clearly achieved our objective. The best compression codec has been found via extensive experiments using four performance metrics. In particular, the combination of PCA and X265 yielded the best performance in most cases, as the performance gain is about $5 \mathrm{dBs}$ in terms of PSNR at 10 times compression. However, from the practical implementation point of view, J2K may be simpler in terms of computational efficiency with only slight performance drop.

\section{ACKNOWLEDGEMENTS}

This research was supported by NASA Jet Propulsion Laboratory under contract \# $80 \mathrm{NSSC} 17 \mathrm{C} 0035$. The views, opinions and/or findings expressed are those of the author(s) and should not be interpreted as representing the official views or policies of NASA or the U.S. Government.

\section{REFERENCES}

[1] Bell III, \& J. F. et al, (2017) "The Mars Science Laboratory Curiosity Rover Mast Camera (Mastcam) Instruments: Pre-Flight and In-Flight Calibration, Validation, and Data Archiving", AGU Journal Earth and Space Science.

[2] Ayhan, B \& Kwan, C \& Vance, S, (2015) "On the Use of a Linear Spectral Unmixing Technique for Concentration Estimation of APXS Spectrum", J. Multidisciplinary Engineering Science and Technology, 2, 2469-2474.

[3] Wang, W., Li, S., Qi, H., Ayhan, B., Kwan, C., Vance, S., (2014), "Revisiting the Preprocessing Procedures for Elemental Concentration Estimation based on CHEMCAM LIBS on MARS Rover", 6th Workshop on Hyperspectral Image and Signal Processing: Evolution in Remote Sensing (WHISPERS)

[4] Wang, W., Ayhan, B., Kwan, C., Qi, H., Vance, S., (2014), "A Novel and Effective Multivariate Method for Compositional Analysis using Laser Induced Breakdown Spectroscopy", 35th International Symposium on Remote Sensing of Environment

[5] Ayhan, B.; Dao, M.; Kwan, C.; Chen, H.; Bell, J.; Kidd, R., (2017), "A Novel Utilization of Image Registration Techniques to Process Mastcam Images in Mars Rover with Applications to Image Fusion, Pixel Clustering, and Anomaly Detection", IEEE Journal of Selected Topics in Applied Earth Observations and Remote Sensing,

[6] Kwan, C.; Dao, M.; Chou, B.; Kwan, L. M.; Ayhan, B., (2017), "Mastcam Image Enhancement Using Estimated Point Spread Functions", IEEE Ubiquitous Computing, Electronics \& Mobile Communication Conference, New York.

[7] Kwan, C.; Chou, B. and Ayhan B., (2018), "Enhancing Stereo Image Formation and Depth Map Estimation for Mastcam Images", IEEE Ubiquitous Computing, Electronics \& Mobile Communication Conference, New York.

[8] Kwan, C.; Larkin, J., (2017), "Perceptually Lossless Compression for Mastcam Images", IEEE Ubiquitous Computing, Electronics \& Mobile Communication Conference, New York.

[9] Haines, R. F.; Chuang, S. L., (1992), "The effects of video compression on acceptability of images for monitoring life sciences experiments", NASA-TP-3239. 
Signal \& Image Processing: An International Journal (SIPIJ) Vol.10, No.1, February 2019

[10] Garrett-Glaser, J., (2010). "Patent skullduggery: Tandberg rips off x264 algorithm," online https://lwn.net/Articles/417562/.

[11] Hruska, J., (2013), "H.265 benchmarked: Does the next-generation video codec live up to expectations?" ExtremeTech.

[12] International Organization for Standardization, "ISO/IEC 15444-1:2016 - Information technology -JPEG 2000 image coding system: Core coding system”, retrieved 2017-10-19.

[13] Ayhan, B.; Kwan, C. and Zhou, J., (2018), “A New Nonlinear Change Detection Approach Based on Band Ratioing”, Algorithms and Technologies for Multispectral, Hyperspectral, and Ultraspectral Imagery XXIV.

[14] Glaser, F., (2010), “First Look: H.264 and VP8 Compared”, Diary of An x264 Developer.

[15] Converse, A., (2015), "New video compression techniques under consideration for VP10", presentation at the VideoLAN Dev Days.

[16] Haykin, S., (1993), "Neural Networks and Learning Machines", Pearson Education.

[17] Wu, J.; Liang, Q. and Kwan, C., (2012), “A Novel and Comprehensive Compressive Sensing based System for Data Compression”, IEEE Globecom.

[18] Blanes, I., Magli, E., and Serra-Sagrista, J., (2014), “A tutorial on image compression for optical space imaging systems”, Geoscience and Remote Sensing Magazine, IEEE, vol. 2, no. 3, pp. 8-26.

[19] Du, Q. and Fowler, J. E., (2007), "Hyperspectral image compression using JPEG2000 and principal component analysis”, Geoscience and Remote Sensing Letters, IEEE, vol. 4, no. 2, pp. 201-205.

[20] Zhou, J. and Kwan, C., (2018), “A Hybrid Approach for Wind Tunnel Data Compression”, Data Compression Conference, Snowbird, Utah, USA.

[21] Kwan, C. and Luk, Y., (2018), "Hybrid sensor network data compression with error resiliency", Compression Conference, Snowbird, Utah, USA.

[22] Strang, G. and Nguyen, T, (1997), "Wavelets and filter banks”, Wellesley-Cambridge Press.

[23] Kwan, C.; Li, B.; Xu, R.; Tran, T. and Nguyen, T., (2001), "Very Low-Bit-Rate Video Compression Using Wavelets", Wavelet Applications VIII, 4391, 176-180.

[24] Kwan, C.; Li, B.; Xu, R.; Tran, T. and Nguyen, T., (2001), "SAR Image Compression Using Wavelets", Wavelet Applications VIII, 4391, 349-357.

[25] Kwan, C.; Li, B.; Xu, R.; Li, X.; Tran, T. and Nguyen, T. Q., (2006), "A Complete Image Compression Codec Based on Overlapped Block Transform”, Eurosip Journal of Applied Signal Processing, 1-15.

[26] Ponomarenko, N.; Silvestri, F.; Egiazarian, K.; Carli, M.; Astola, J. and Lukin, V., (2007), “On between-coefficient contrast masking of DCT basis functions", Proc. Third International Workshop on Video Processing and Quality Metrics for Consumer Electronics, Scottsdale, AZ, USA.

[27] Kwan, C.; Shang, E. and Tran, T., (2018), "Perceptually lossless image compression with error recovery", 2nd International Conference on Vision, Image and Signal Processing, Las Vegas, NV, USA.

[28] Kwan, C., Shang, E. and Tran, T., (2018), "Perceptually lossless video compression with error concealment", 2nd International Conference on Vision, Image and Signal Processing, Las Vegas, NV, USA. 


\section{Authors}

Chiman Kwan received his Ph.D. degree in electrical engineering from the University of Texas at Arlington in 1993. He has 15 patents, 52 invention disclosures, 320 technical papers in journals and conferences, and 450 technical reports. Over the past 20 years, he has been the PI/Program Manager of over 115 diverse projects with total funding exceeding 36 million dollars. He is also the founder and Chief Technology Officer of Signal Processing, Inc. and Applied Research LLC. He received numerous awards from IEEE, NASA, and some other agencies.

Jude Larkin received his B.S. in Computer Science from Franciscan University of Steubenville in 2015. He is a software engineer at ARLLC.

Bence Budavari received his B.S. in Audio Engineering from Belmont University in 2015. He is a software developer at ARLLC.

Bryan Chou received his B.S. in Electrical Engineering from the George Mason University in 2015. He is an electrical engineer at ARLLC. 